\title{
Health Disparities Among Health Care Workers
}

\author{
Barbara Mawn ${ }^{1}$, Eduardo Siqueira ${ }^{1}$, Ainat Koren ${ }^{1}$, Craig Slatin ${ }^{1}$, Karen Devereaux Melillo, \\ Carole Pearce $^{1}$, and Lee Ann Hoff ${ }^{1}$ \\ ${ }^{1}$ University of Massachusetts Lowell, Lowell, Massachusetts, USA
}

\begin{abstract}
In this article we describe the process of an interdisciplinary case study that examined the social contexts of occupational and general health disparities among health care workers in two sets of New England hospitals and nursing homes. A political economy of the work environment framework guided the study, which incorporated dimensions related to market dynamics, technology, and political and economic power. The purpose of this article is to relate the challenges encountered in occupational health care settings and how these could have impacted the study results. An innovative data collection matrix that guided small-group analysis provided a firm foundation from which to make design modifications to address these challenges. Implications for policy and research include the use of a political and economic framework from which to frame future studies, and the need to maintain rigor while allowing flexibility in design to adapt to challenges in the field.
\end{abstract}

\section{Keywords}

case studies; disparities, health care; health care, workplace; occupational health; workplace

In 1999, the National Institutes of Health issued a request for proposals that sought "to foster multi-disciplinary research that would elucidate the underlying mechanisms by which the interaction of social and physical environments leads to health disparities." In response to this request, researchers at the University of Massachusetts Lowell developed a proposal entitled "Health Disparities Among Health Care Workers." The National Institute of Occupational Safety and Health (NIOSH) funded this study from 2000 to 2006. In the early phase of the research, the team established a project name for the study: Promoting Health and Safety in Employment in Health Care (PHASE in Health Care).

The study design had multiple components, including a case study of the political economy of the work environment in various health care sectors. The PHASE team conducted the study to understand how the prior 2 decades of health care system restructuring in New England shaped the organization of the work environment, employee diversity support, and

Reprints and permission: http://www.sagepub.com/journalsPermissions.nav

Corresponding author: Barbara Mawn, University of Massachusetts Lowell, Nursing, 3 Solomont Way Suite 2, Lowell, Massachusetts 01854,USA, barbara_mawn@uml.edu.

Declaration of Conflicting Interests

The authors declared no conflicts of interest with respect to the authorship and/or publication of this article. 
the nature of occupational injury disparities. The case study incorporated primarily qualitative data from document review, focus groups, and key informant interviews. In addition, the researchers analyzed quantitative demographic and injury data, which helped to provide a baseline of employee characteristics.

In this article we describe the case study research design and explore the barriers and constraints experienced during the implementation of the study that necessitated design flexibility and modification. A recent review of published qualitative research by Shin, Kim, and Chung (2009) identified that case study methods have rarely been reported in two major qualitative journals in comparison to other methods such as grounded theory and phenomenology. Thus, although this article does not include the final results of the study, we share the knowledge gained on the complexity of implementing various facets of the lesscommonly reported case study design, with examples of its impact on some of the preliminary study findings.

We anticipated that conducting field research in arenas of social conflict would lead to ongoing modification of research methods and strategies. These social conflicts included the control over workplace conditions where measures to control health care costs collided with the requirements to provide an appropriate degree of patient care. However, beyond our expectations, we learned the difficulties of study recruitment when researching a work environment that strongly relies on contingent employment arrangements. These arrangements include part-time employment; unanticipated, intermittent reductions of work hours; and limited employee control over work assignments and scheduling.

Since the mid 1980s the U.S. health care system has evolved, resulting in unique occupational health and safety risks for its workers. Nonfatal injury rates in the health care sector were negligible up through the mid-1980s. By the mid-1990s however, they increased substantially, whereas nationwide, occupational injuries for almost all other industries remained stable (Shindul-Rothschild, Berry, \& Long-Middleton, 1996). In 1995, the incidence rate of injury and illness in the hospital workforce was 10.1 cases per 100 fulltime employees and 18.2 per 100 in nursing and personal care facilities, compared to a total private-sector rate of 8.1 per 100 (U. S. Bureau of Labor Statistics, 1997).

Key health care sectors (hospitals, long-term care, and home care) employ a workforce that has substantial variability in terms of socioeconomic status (SES), gender, ethnicity, and work-related exposures. The sectors have undergone 2 decades of restructuring, using externally imposed cost-control measures (Jessop, 1992), and work reorganization models (Brannon, 1994; Landsbergis, Cahill, \& Schnall, 1999; Weinberg, 2003).

\section{Theoretical Framework}

The PHASE team designed this study to explore health care work environments within the framework of their political economy. Such analysis assumes that political and economic forces shape civil society and the actors within it. Therefore, a political economy of the work environment locates political and social actors as agents within a larger economic context (Levenstein \& Tuminaro, 1991; Wooding \& Levenstein, 1999). 
We established propositions from our research questions and framed them within specific dimensions of the political economy of the work environment (Levenstein \& Tuminaro, 1991; Levenstein, Wooding, \& Rosenberg, 1995; Siqueira, 1998; Slatin, 1999; Wooding \& Levenstein, 1999). These dimensions are ideology and market dynamics; technology; political and economic power dynamics; and demographics and labor markets. We developed a complex onion model to include these dimensions within five levels of the organization of the political economy. Figure 1 depicts a simpler version of our conceptual model, which depicts five organizational levels that impact health care organizations' policies and ultimately the health and safety of workers.

\section{Ideology and Market Dynamics}

Nearly 3 decades of neoliberal ideologies of free markets and greatly restricted government social interventions and associated economic and industrial organization have resulted in a restructuring of the U.S. health care system. Consequently, health care facilities/institutions function increasingly as competitive and market-based organizations. An inherent contradiction is established between this mode of operation and a more historical mission of the health care system to serve the health needs of communities.

\section{Technology}

This restructuring is accelerated by, and also demands, changes in technology that support a degree of automation of service delivery and downsizing of the service work-force.

Additionally, changes in population health status and the political economy of the health care work environment result in new sets of health and safety risks and outcomes, such as blood-borne and other infectious pathogens and musculoskeletal disorders associated with lifting and repetitive motion hazards. The use of new employee protection technologies should be associated with the level of risk and injury/illness experienced by the workforce.

\section{Political and Economic Power Dynamics}

Power dynamics within the United States establish control of the health care system by a set of payers - primarily insurance companies, care management companies (e.g., health maintenance organizations), and employers who pay for the health insurance of their employees. Additionally, the federal and state governments are significant payers, who act independently to reduce their costs, but who also influence other payers to adjust costs. Labor unions also have power within this system, particularly regarding government regulations and management of health care workforce policies and procedures.

\section{Demographics and Labor Market}

Shifts in the size of the incoming health care workforce, along with an existing aging health care workforce (particularly nurses), have established the basis for changes in staffing levels and ratios in health care provider organizations. Subsequently, there is reallocation of job tasks, an increased introduction of less-skilled workers, and in many locations, a reliance on low-wage minority and immigrant workers. 


\section{Methods}

\section{Study Design}

Case study researchers explore conditions and phenomena to provide in-depth explanations of the context and history of organizations and institutions (Ragin, 1999; Sofaer, 1999; Yin, 1999). Case study research is often built on sets of propositions (Yin, 1994, 1999). Case study researchers seek to understand a contemporary political phenomenon in which the researcher has no control over the event or case being studied (Yin, 2003). This method allows for triangulation of data and flexibility in the procedures for data collection to allow for the incorporation of new data sources and alternate means of gathering data (Eisenhardt, 1989).

We defined the case or object of our study as the health care industry in New England. Our empirical evidence for the case was drawn from nested case studies of two community hospitals, two long-term care facilities, and data drawn from focus groups with members of two health care unions.

Stake $(1995,2005)$ has suggested that case study research is both intrinsic and instrumental with the goal of gaining in-depth understanding that broadens the researchers' perspective of specific situations. It is instrumental if it is representative of a population of concern. Yin has suggested that case study research is considered revelatory if the unit of study is not entirely representative of the intended population but can give insight into similar cases (1994). We designed this case study to be instrumental, providing a deeper understanding of the context for health disparities among health care workers, at least in the New England region of the United States. Given the research constraints encountered, the final results can be considered revelatory, as they allow for analytical generalizations, hypothesis generation, and intervention recommendations despite the lack of full representation from all types of health care organizations.

In contrast to empirical or statistical generalizations drawn from quantitative research, analytical generalizations include reasoned judgments that allow one to guide other similar cases based on the results of the case study inquiry (Hamel, Dufour, \& Fortin, 1993; Kvale, 1996; Sharp, 1998; Yin, 1994). This in-depth analysis of workers from varied institutions and agencies allowed a window of opportunity to explore the issue of health care worker safety from a broad environmental and social perspective. We prospectively collected data from the participating sites from 2001 to 2005 . We decided, however, to expand the case study time period to the 10 years prior to the PHASE project to elicit an historical context; thus, the study was bound in time from 1990 through 2005. Data collected prior to 2001 included historical documents, such as relevant regional policies and regulations, internal facility reports, facility newsletters, and publicly available media reports. The institutional review boards of the participating hospitals, nursing homes, unions, and/or the University of Massachusetts Lowell approved our original application and subsequent revisions to the protocol. 


\section{Study Aim}

The aim of the PHASE in Health Care study was to examine physical, social, and behavioral risks and the complex pathways that produce health and illness in the health care workforce. We investigated occupational injuries across the socioeconomic gradient. Our team conducted this research at facilities experiencing the disruptions of health care system restructuring, creating a difficult setting for conducting occupational safety and health research. The PHASE team investigated how this restructuring shapes facility-level decision making about health care worker health and safety and workforce diversity support, as a way to determine the extent of health disparities and to understand the bases for them.

\section{Propositions}

The case study team established five research propositions based on the theoretical framework:

1. The macro-level political economy shapes the relations between the various health care system actors found within a specific health care provider organization.

2. The relations between the various health care system actors would establish the micro-level political economy at the point of production, i.e., point of service.

3. The political economy of the health care work environment will establish the context for the nature and extent of health disparities within the health care workforce.

4. The macro- and micro-level political economies of the work environment will determine the specific nature of and pathways toward occupational injury disparities. Occupational injury disparities cannot be assumed to be based exclusively within either a race/ethnicity, class, gender, age, or other categorical context.

5. The organization of diversity support in a work environment will be a mediating factor determining the pathways toward occupational injury disparities. Diversity support within a provider organization encompasses the inclusion of workers from varied racial/ethnic/gender backgrounds. It is influenced by social norms and biases.

\section{Study Team}

The PHASE in Health Care research study team included the disciplines of epidemiology, ergonomics, nursing, work environment policy, economics, health education, anthropology, and community psychology. A multifaceted design included four components: case studies that served to analyze the political economy of each participating facility's environment; epidemiological surveys to collect employee baseline and follow-up data to examine workrelated injuries and illnesses; workplace observations and exposure assessments; and a community outreach and education program (COEP). We created research teams to plan and conduct field activities related to these four components. The COEP allowed us to share information about the project with the communities of interest, including health care 
professionals, union members, academic researchers, government officials, and participating site employees.

Within the case study team of the PHASE in Health Care project, researchers from the numerous disciplines noted above contributed to the study using mixed methods approaches, with qualitative methods being the predominant approach for this case study. O'Cathain, Murphy, and Nicholl (2008) suggested that "methodological respect" and a principal investigator who demonstrates commitment to the value of integration of mixed methods is the key to achieving successful teamwork. Although the PHASE case study team did lose some of its original core members over the course of the study, it was able to integrate a successful interdisciplinary approach during all phases of the research process (Slatin, Galizzi, Melillo, Mawn, \& the PHASE in Health Care Research Team, 2004). Despite potential challenges confronting collaborative research among multiple disciplines using more than one research paradigm (Austin, Park, \& Goble, 2008; Cheek, 2008; Mendlinger \& Cwikel, 2008), as Morse (2008) suggested, we did not shortchange one approach for the other; the team fostered and maintained mutual respect.

\section{Protocol Guide: Data Matrix}

The case study research team developed a case study data collection matrix as a preliminary step in the research process to serve as the framework for study protocols and data collection. The matrix was a written document that included a detailed description of research questions and potential data sources to answer them. Development of the matrix provided the team with an opportunity to pose questions, identify indicators of conditions under investigation, plan data-collection strategies, review methodological options, and build consensus about the units of analysis (Miles \& Huberman, 1994). We organized the matrix by seven aspects of the political economy of the health care work environment derived from the study's theoretical framework. These included characteristics of the organization, labor/ management relations, organizational hierarchy and structure, workforce characteristics, pay structures and benefits, employee health and safety policies and practices, and technology.

Within each category of the data matrix, we identified key indicators that we would explore across sites to examine patterns and processes that can impact health disparities and worker health and safety. Table 1 exemplifies this level of detail in one of the matrix categories. The matrix was the foundation for our data-organization scheme and guided our analytical process. It served as the basis for our sampling plan, data-retrieval plan, coding structure for data organization, and analysis. Using the matrix to map data collection enhanced the team's ability to reliably conduct such a complex study. The matrix development process helped to bind the case in both time and place (Creswell, 2006; Stake, 1995).

Our matrix development process was particularly helpful in deciding how best to study disparities. Health disparities between segments of the population likely result from social inequalities and institutionalized discrimination. Our collective experience was that few people can easily discuss the existence and consequences of such discrimination in their organizations. So, we decided that rather than asking questions about discriminatory practices that might result in health disparities between groups of employees, we would use diversity support as a proxy. Although we realized that these are not the same, we believed 
that our informants would be more likely to provide us with strong clues about differential employee support practices if we focused on diversity issues rather than asked about discriminatory practices.

\section{Recruitment of Community Partners}

The original study plan was to conduct the research in varied health care settings with workers from diverse ethnic backgrounds. Commitments were obtained from two acute care, nonprofit community hospitals; a state hospital dedicated to long-term care; three nursing homes owned by a large corporate chain; and a local home health care agency. We intended to include facilities with and without union representation of employees. An early study challenge was to find partnering organizations that would allow the team access to internal reports and documents, managers at various levels within the hierarchy, and the workers themselves. We experienced several early facility withdrawals from the study. The home care agency withdrew its cooperation with the stated concern that published study findings could be used to organize the agency's employees into a union. The one committed hospital with union contracts withdrew because of problems unrelated to the study, resulting in the loss of a substantial number of union member participants. Three facilities of a national corporate nursing home network withdrew from the study following instruction from the corporate office (located in another state). Although facility managers had agreed to participate, this corporation was unwilling to permit their facilities' personnel to give the time and effort needed. A union represented the certified nursing assistant employees of one of the three facilities. Withdrawal of that facility resulted in the total loss of facilities with union workers.

Later in the study, one of the remaining two hospital sites withdrew from the study, even though we had commenced with considerable case study research data collection. A new management team made the decision after managerial downsizing, which resulted in labor/ management tensions. The hospital's newly hired human resources director was unwilling to risk adding to these tensions by having employees engage in research activities that might result in heightening their awareness of workplace health and safety concerns. The hospital discontinued participation in the study, limiting collected data to demographic and injury reports, facility policy documents, and interviews with a limited sample of administrators, managers, and supervisors. At that point, management denied access to workers for focus groups, although they permitted the use of data collected to that point.

After these initial setbacks, two additional nursing homes agreed to participate in all components of the proposed case study research. To maintain anonymity we labeled the participating facilities Hospital 1 (no direct worker data), Hospital 2, Nursing Home (NH) 1, and $\mathrm{NH} 2$. NH1 is part of a national, for-profit corporation and $\mathrm{NH} 2$ is a nonprofit facility with religious affiliation. Both nursing homes and Hospital 2 agreed to participate in all research components. Each partnering organization assigned a manager as a project liaison.

With the loss of facilities with union contracts, the PHASE team acknowledged that the available data from participating sites would lack the potentially unique perspective of unionized workers. To include these views, the team decided to approach two unions to request collaboration with the project. Two unions representing health care workers agreed 
to allow us to conduct focus groups with their members who were employed at various health care facilities. These are referred to as Union 1 and Union 2. Although the data would not be specific to participating facilities, we believed that we would be able to learn a great deal about health care sector health and safety issues from these workers. Union members worked in facilities that were located in eastern Massachusetts, including mid- to large-size hospitals in urban and suburban settings.

\section{Individual Study Participant Recruitment}

An original goal was to obtain data from nonunionized and unionized health care facilities and to include employees with diverse racial/ethnic backgrounds and varied occupational roles within each facility. Our case study sampling was aimed at drawing a sample representative of the health care workforce employed in New England facilities. We correctly presumed that the workforce in our participating hospital sites, however, was not as diverse as that in Boston and other major urban centers. Urban settings tend to have a higher proportion of workers of color and those who do not speak English as a first language. Thus, our sample was limited in its representativeness. Nonetheless, we did recruit minorities in the nursing home sites, and our final sample likely reflected the demographics of much of the New England health care workforce outside of the major urban centers.

Given the variation in the organizational structure and physical attributes of the participating study sites, we developed recruitment protocols that best fit the conditions and needs of each site. Employee preferences determined recruitment mechanisms and included general inhouse email invitations, on-site postings and advertisements for the study, individual letters delivered on site, on-site recruiters in the cafeterias, and direct telephone calls to individuals selected as interview candidates. Response to recruitment efforts differed at each site. For the union focus groups, each union's project liaison recruited participants through one-onone work contact, mailings, and email notices and/or telephone calls. Recruitment at the hospitals and nursing homes required unique perseverance and, ultimately, flexibility in the method adopted.

Recruitment for interviews was easier with administrators than with front-line workers. At most sites, there was no conflict with the administrators' time for the interview; it was assumed as a job duty. Only one of the facilities, however, would permit nonmanagerial employees to leave a work shift for focus group participation. Although this was largely because of tight staffing levels that restricted department supervisors' scheduling flexibility, it also demonstrated employers' limited commitment to the study objectives, as well as the different levels of autonomy afforded managerial and nonmanagerial personnel. The research team scheduled focus groups before or after shifts or during lunch breaks to facilitate employee participation.

Generally, focus group research uses purposive participant selection, in the interest of attaining information from those who have experience with the identified topic of interest (Creswell, 2006; Denzin \& Lincoln, 1998; 2005; Kitzinger, 1994; Krueger \& Casey, 2000; Morgan, 1993, 1997, 1998; Patton, 1990; Rice \& Ezzy, 1999). An imbalance of power generally is found in U.S. workplaces where employers, but not employees, have the power and legal right to control the conditions of work and employment, use of financial resources, 
and determinations of acceptable risk (Ashford \& Caldart, 1991; Berman, 1978; Braverman, 1974; Levenstein \& Tuminaro, 1991; Levenstein et al., 1995; Noble, 1986; Wooding \& Levenstein, 1999). Because the facilities participating in our study were not unionized, we could not ask a union leader or an employee representative to identify employees best able to provide data in focus groups. To avoid potential bias from relying on a manager to supply us with potential worker participants, our initial plan for the selection of focus group participants was to recruit from a randomized list of employees from various departments.

We implemented our initial focus group participant recruitment strategy in Hospital 2, using both a purposive (selected departments based on key informant interview data) and a stratified randomized approach (stratified by department and shifts, using employee lists supplied by the facility). This method of recruitment resulted in very few responses. To increase study participation among workers we then recruited more broadly across departments within the hospital. We learned from the few initial worker study participants that the advertised time frame (70 minutes) was too long despite the incentives given, and that a commitment in advance was difficult for many workers given their family and other commitments.

The case study team conducted 60 key informant interviews with employees who worked in mid- to high-level management positions at the four participating health care facilities. These included the chief executive officer, nursing directors, nurse managers, employee health director, safety and security directors, and administrators from various other departments within each facility. The case study researchers also interviewed human resources and pastoral care managers in some participating facilities. We used the interviews to understand management's awareness of and attitudes toward the key theoretical components identified in the matrix, as well as to learn about facility policies and practices regarding workplace health and safety and diversity support. In addition, the team learned about the history of each facility, which assisted in the development of focus group priority topics.

The case study team researchers conducted facility-based focus groups with 137 workers who did not have a management position. These study participants included those involved with direct patient care (e.g., nurses, nursing assistants, $x$-ray technicians) and those whose jobs did not primarily involve direct patient contact (e.g., housekeeping, food services, and clerical employees). We additionally interviewed 56 union workers in 9 focus groups, for a total of 193 workers as study participants. The unionized workers included nurses and nursing home patient care assistants (PCAs). These direct and nondirect patient care workers from the hospitals, nursing homes, and unions generously shared their personal knowledge, experience, and stories. They also provided suggestions to review reports and formerly unidentified policies, identified other informants to interview, and suggested additional operations/processes to observe. The data they provided were critical to understanding the sociopolitical and economic contexts of health and safety disparities related to morbidity associated with workplace exposures. Table 2 identifies the numbers of managers and workers who participated across the various sites. 
From the lessons learned at Hospital 2, we changed the recruitment strategy at the nursing homes by making a direct invitation to all potential workers, rather than attempting to randomize the sample. The smaller size and structure of the nursing homes in comparison to the hospital facilitated the recruitment effort as well. The study team recruited union members for focus groups in coordination with a union liaison who was paid by the study to organize focus groups. The Union 2 liaison was a union representative to two nursing homes unaffiliated with the study. The Union 1 liaison was a union member who was also a doctoral candidate at the University. Both of these individuals were largely responsible for the success of these focus groups. The focus groups with Union 1 members were our only sessions that fully followed our original protocol designs in terms of conducting focus groups on targeted topics vs. general health and safety concerns.

\section{Data Collection}

The team members collected data from each of the four health care facilities as well as from the two union groups. One researcher coordinated the team's research at each site/facility and with each union. The study aims and questions required gathering data from a multitude of sources and perspectives. Data collection was an iterative process that began with an initial walk-through observation-a guided tour of the participating facilities-for quick and summary observations of their major units. The observations helped to form the basis for developing a data collection plan. They also informed us of the likely key informants to interview and how we could begin to frame focus group topics and participant recruitment. We used each stage of data collection to determine new data needs and assess the strength of existing data.

Data sources at each facility included documents and archival data, interviews with managers and workers, facility policies, site observations, and media reports about the facility. We also obtained demographic data, labor/management and other policies, wage and benefit program information, staffing data, organizational charts, injury records (Occupational Safety and Health Administration logs and Workers' Compensation system reports), health and safety program information, and return-to-work policies and program information. Additional data included annual audit reports, financial balance sheets, and other operational reports. We obtained meeting minutes and information about various committees, such as health and safety or cultural competence, if they existed. A key document data source at the hospitals was the employee newsletter, including 10 years' worth of weekly newsletters at one hospital, which provided published employee concerns and management responses. The newsletters reported the strains resulting from health care system restructuring, and the ways that both labor and management reacted to those strains. They also detailed the ways in which each organization's philosophy of care was integrated into reorganization strategies. These data were particularly helpful for exploring the context within which occupational injuries occur, and sometimes the conditions leading to specific types of injuries.

The team originally planned focus groups as 60-minute sessions preceded by 10 minutes for introductions, review of ground rules, and measures to protect confidentiality. We provided food, as well as a $\$ 25$ cash incentive. We set focus group size with a four-person minimum 
and eight-person maximum. The team developed scripts for five topic areas: workplace injuries and health and safety practices, programs, and policies; postinjury return to work; diversity support and cultural competence; workplace violence; and health care system restructuring and reorganization. We identified specific job categories for recruitment to each focus group. The case study team initially targeted and recruited employees from populations of color or for whom English was a second language for sessions about diversity support.

\section{Data Analysis}

Two or more research team members-usually representing different disciplines or fields of expertise within a same discipline-conducted small group analysis. Using this small group approach, we established analytical triangulation, a process previously described in the literature for use in hospital-based case study research (Thomas, Blacksmith, \& Reno, 2000). Analyst triangulation reduces single-person analysis bias as well as bias inherent in a single-discipline orientation (Patton, 1999; Yin, 1994). The case study research team organized and analyzed the data reports (summaries, transcripts, field notes, and memos) with the Qualitative Software Research (QSR) NVivo 2 computer software program (Bazeley \& Richards, 2000; Richards, 1999).

The case study team used categorical and thematic codes that we established in our data collection matrix. We modified these established, clearly defined codes only by group consensus. The NVivo software program (Bazeley \& Richards, 2000) allowed us to map thematic codes (called nodes in this program) which we could then make into a visual display posted in the computer laboratory for easy reference. We clearly defined each major category/node and subsection to ensure consistency over the 5-year period during which changes in team composition occurred. Data that did not fit any of the predetermined thematic nodes were categorized and mapped separately for further analysis to determine their relevance to the study. One example of such data was subsequently coded as the "culture of nursing," which later played a key role in understanding an important aspect of the health care work environment. The small group process enhanced the iterative process of coding and code revision based on field experience and data analysis described by Miles and Huberman (1994). When new themes emerged, which indicated the need for additional data, the researchers asked the informants for clarification, conducted further interviews, and/or acquired new documents.

\section{Subsequent Modifications of the Research Design}

We studied four facilities that were under stress because of the ongoing health care system restructuring, with conditions such as changing regulations, shifts in reimbursements, the need to reduce labor costs through increased employment of less-trained workers, and decreased employment of highly trained workers such as nurses (the latter was not the case in nursing home settings because the state regulates nurse staffing patterns in long-term care settings). The strains on the facilities required flexibility in how we implemented our research design. In this section we discuss the conditions encountered and the modifications made to our methods. 


\section{Hospital 1}

The team secured early and strong managerial support through a facility liaison who was the senior assistant to the vice president of operations. As this was the first site in the case study, we intended to pilot our design and then modify it as necessary for implementation at the other sites. In this facility we identified the types of documents and data sources likely to be available in a hospital. We had easy access to upper and middle management, as well as consultants from a workers' compensation management firm contracted by the facility. As noted, we completed nearly all data collection from management and document sources; we did not gain direct access to workers because of concerns from new management. At the start of the study, several employees felt encouraged by the presence of the researchers to ask their managers questions about specific facility practices and policies. This placed enough strain on the new management to withdraw from additional data collection involving workers. We did manage to collect limited but useful data regarding employee concerns from 10 years of weekly employee newsletters.

\section{Hospital 2}

The majority of Hospital 2 managers and administrators readily responded and participated in individual key informant interviews. As noted, initial recruitment at Hospital 2 for nonmanagerial workers proved more difficult. Few hospital employees responded to our initial invitations and subsequent recruitment flyers. This hospital had undergone several reorganization processes. One employee told us that other employees were wary that the university researchers were just one more team of organizational change consultants. Some employees, we learned, thought that we were working with management and that information provided to us could be relayed to management and used in a disciplinary fashion against employees. We learned of these barriers by gaining the trust of a few employees and managers. In response, we expanded our Community Outreach and Education Program (COEP) to address these concerns and to build relationships with employees of Hospital 2. The COEP team provided project information to facility personnel in friendly and easy-to-understand formats. We set up an information table at the hospital entrance and the cafeteria. COEP team members explicitly told employees that the PHASE research team was independent of hospital management. Although these measures helped increase study participation, we did not sufficiently overcome recruitment barriers to fully follow the original research design.

Our focus group recruitment flyers provided information about the topics for discussion, the location and time, the incentive award, and whom to call if an employee wanted to participate. Although a few workers responded, we did not secure enough from each category to conduct job-based groups. Thus, as noted, recruitment procedures changed to include an open invitation for all workers at specific times and locations, focusing on a general discussion of the health and safety of workers.

After considerable modifications of recruitment strategies and data collection methods to address these challenges, we were able to recruit 81 nonmanagerial study participants at Hospital 2. Changes included alternative site locations (one Hospital 2 nurse volunteered her home to discuss the issues with her coworkers) and reducing the time commitment of the 
focus group to 30 minutes. Another successful site strategy was the use of an easily accessible conference room located outside the hospital lobby for open forum discussions during workers' 30-minute lunch breaks, with a free lunch. Two such events were held, with more than 30 people attending each. Although we advertised a 30-minute timeframe to elicit participation, these forums actually went over the time limit because of the participants' enthusiasm and engagement in the discussion. Facilitators asked several key questions from all five of our original targeted focus group scripts. Instead of workers responding to the drafted questions from the original focus group topic guides, they responded to some key questions from each topic set. Although this method of a large group forum vs. a small focus group did not allow for in-depth discussions, it did allow a venue for workers to express their major concerns. Two members of the research team attended the individual interviews, small group interviews (less than 6), focus groups, and large forum sessions, with one conducting the interview and the other observing and taking notes about the process and dynamics of the interviews. The team audiotaped all interviews and transcribed them verbatim.

\section{Nursing Homes 1 and 2}

Given the lessons learned at the hospital site, we implemented our COEP efforts early on in the two nursing homes to build relationships with managers and employees prior to commencing data collection. Nonetheless, we faced similar, though not as severe, constraints in available time for nonmanagerial employee participation and the number of employees willing to participate. We were able to conduct focus groups according to our protocols for length, but we had to combine topics because we were unable to recruit sufficient employees for separate focus groups.

The case study team implemented previously described protocols and plans for data collection at both nursing homes, but here again, site-specific conditions required some modification. The nursing homes had a more diverse workforce than the hospitals, reflecting the stronger reliance on immigrant workers. We conducted several focus groups on diversity and cultural competency in the workplace in each facility; each group included ethnic/racial diversity. Culturally and linguistically competent translators/research staff conducted these focus groups with Brazilian and Haitian employees, who spoke Portuguese and Creole respectively, and with Spanish-speaking employees who were mostly from Central America. The focus groups on the remaining topics generated data about diversity issues because they included employees representing ethnic, racial, and job-category diversity. Focus groups at $\mathrm{NH} 2$ were shorter than the time established by protocol. At most they lasted 1 hour, with employees attending during a 30-minute lunch break. In a unique circumstance, the NH2 management allowed employees to use 30 minutes of work time for focus group participation. However, few employees felt that they could afford to take that time from their demanding workload.

\section{Union Members}

Focus groups with workers outside of our participating facilities included registered nurses from Union 1 and certified nursing assistants who were members of Union 2. These focus groups could not provide direct, observable data by the study team about the conditions in 
the union members' places of employment. However, they provided rich data regarding the perceived work environment conditions, policies, and practices in a broader set of health care provider organizations (including nursing homes and a variety of hospital settings). These data included perspectives about the context of health care work and how health care system restructuring had shaped the work environment. It was useful to contrast these data with the data from the participating hospital and nursing home sites, to assess the strength of our propositions.

Union 1 nurses participated in seven focus groups that covered all of the original designated topics, in full accordance with our original protocol. Few participants were people of color, all spoke English as their first language, only a few were men, and many were union activists. This union had a strong history of health and safety activism. Many participants were not only quite prepared to discuss the focus group topics with us, but had experiences of organizing around these issues or actually working with management to establish strong health and safety programs. These focus group participants also were much more versed than their nonunion nursing counterparts from the participating study sites in understanding the political and economic dimensions of health care system restructuring and how it shapes policies at the facility level. Thus, they provided us with a contrast to the views of workers from our other sites.

\section{Rigor}

Denzin and Lincoln (2005) identified various philosophical underpinnings related to evaluating rigor in qualitative research. In an earlier work, Lincoln and Guba (1985) proposed four major criteria for rigor or "trustworthiness" that set the standard for the evaluation of qualitative research. These criteria include credibility, transferability, dependability, and conditionality.

PHASE Case Study Team techniques to establish rigor included training in qualitative methods for all members. The study team participated in numerous meetings and workshops to learn how to conduct qualitative interviews and focus groups, qualitative data analytic methods, and data-management strategies using the NVivo software program (Bazeley \& Richards, 2000). Also, the team collectively developed definitions for all initial codes to maximize analytic dependability across the group. We established and rigorously applied a dependable and confirmable audit trail. The maintenance of reflective journals and field notes for data collection and analysis logs strengthened data confirmability. In addition, the development of protocols to support consistency and reliability in the collection and organization of data across sites enhanced rigor and facilitated common formats for data analysis (Stake, 1995; Yin, 1994). Protocols included scripts for key informant interviews and focus groups and guides for the organization and management of collected data. All researchers participated in focus group facilitation training to assure consistent protocol compliance. Other protocols included procedures and formats for writing reports, memos, field notes, summary documents, and naming conventions for all such documents, as well as interview and focus group transcripts, to facilitate consistency across a large research team. 
The team audiotaped all interviews, including individuals, focus groups, and large forums, with permission. An outside company transcribed the interviews with no personal identifiers on the tapes or transcripts. Researchers wrote pre- and postinterview and focus group notes to provide an audit trail and incorporate these observations and insights into the data analysis process.

Triangulation in data collection and analysis processes provided credibility and dependability. Triangulation of collected data supported our ability to contrast interview and focus group data against documents, administrative policies and records, and observational data. Patton (1999) and Yin (1999) discussed how qualitative data triangulation supports understanding differences and contradictions that emerge through analysis.

We examined the transferability and conditionality of our analyses as we compared findings across study sites. Site-specific research in both hospital and long-term care settings enhanced our understanding of their responses to market pressures and population health trends. The conditionality based on employee demographics and management attitudes, policies, and practices regarding race and ethnicity demonstrated the need for investigation of disparities at the facility level. For example, the hospitals employed a less racially and ethnically diverse workforce, but had resources and some management imperatives to establish cultural competency programs for their employees. The nursing home sites had much greater workforce diversity, but fewer management resources to understand how best to provide diversity support. These differences could account for some of the variations in themes across the two types of facilities.

\section{Discussion}

Health research in dynamic community and workplace settings inherently presents potential challenges related to data access and management cooperation. Our difficulties in recruiting workers to participate in focus groups forced modifications of some study protocols. Lacking sufficient participants for focus groups, we recruited individual workers for interviews (a data collection method originally intended only for administrators) and also held large group forums as venues for data collection. The large group forums had more than 30 attending employees at times, making it difficult to focus the discussion and examine issues raised in depth. We modified the focus groups in the nursing facilities from our original design. We found that these modifications to ensure front-line worker participation assisted in the identification of one of the key preliminary findings: the different perspectives from workers vs. administrators/managers on health and safety risks in the health care setting and procedures in place to prevent injuries.

The impact of the culture of nursing on health care worker health and safety emerged as an example of an unanticipated finding. Although the theory of the political economy of the work environment does account for social and professional cultural norms, the nursing profession's culture was not identified at the outset of the study as a contributing factor to the context of health disparities among health care workers. Nurses talked about their love of the job despite its inherent risks related to the common experience of back strain and other harms related to occupational exposures. As one union nurse shared, "We put ourselves in 
harm's way. ... Adaptation is a terrible thing; you do it because it's expected of you. And eventually you don't even realize how bad it is for you."

The study of social organizations under stress is critical to our understanding of the strengths and limitations of their functioning, even as the research of existing conditions might compromise study design. As researchers, we must be flexible yet rigorously honest in stating the actual and potential limitations resulting from modifications to established protocols. One example of the advantages to our flexible design, necessitated by several original collaborators opting out of the study, was the wealth of information gleaned from the focus groups on workplace health and safety conducted with the union employees. One interesting difference between the union workers and those in our participating nonunion facilities was that the union members concurred that employees in their institutions would usually report all injuries. In contrast, participants from the participating sites told us that there were often barriers to reporting injuries. Indeed, workers in nonunionized facilities often shared their fear of repercussions if they reported an injury. Many workers and managers complacently acknowledged injury as "part of the job," and thus, even though policies existed for the reporting and treatment of serious injuries, the implementation varied and the focus on the prevention of occupational injuries was less visible.

Another finding that emerged in the preliminary analyses included the relationships between health care workers' adverse, work-related health outcomes and the health and status of the patient populations. Commonly, both workers and managers identified the challenges related to the rise of obesity on the increased risk of back and neck sprains among workers.

Similarly, participants noted an increase in violence toward front-line workers from patients. Managers and workers associated this patient-generated violence against workers with the lack of adequate community psychiatric facilities, leading to an increase in psychiatric admissions to the emergency department and medical/surgical wards. Although these findings were not a surprise to some of the team researchers with clinical experience, the team had not initially identified this relationship as a research proposition. The identification of this trend reflects the strength of the applied research methods, which allowed new or "out of the box" themes to emerge.

The field research challenges that we encountered are often an aspect of work environment studies, especially when attempting to collect data from workers within the constraints set by the employers. Some research barriers resulted from the balance of political and social control and power in the workplace (union vs. nonunion, labor vs. management). Other barriers arose from the earlier described contingent working arrangements that have the consequence of limiting workers' time and sense of freedom to participate in a research study. Another set of barriers, in some instances, was mistrust of researchers, expressed by both nonmanagerial workers and managers. Some managers suggested that the research could disrupt the workflow and, at worst, stir up employee dissention with concerns about hazardous working conditions. We did not anticipate some of these barriers prior to the implementation of our study. To conduct and complete the study, we regularly faced problems and obstacles that required the flexibility to modify our original protocols. 
Decisions to modify protocols can reduce the trustworthiness of the data. The methodical and often tedious process of developing a data collection matrix and sets of research protocols provided a solid basis for decisions about design and method modifications. These tools provided a blueprint from which we could assess the implications of any modifications for the strength and viability of the research. The case study research method enhanced our understanding of how health care worker health and safety and diversity support are shaped by the ideological and organizational restructuring of the health care industry.

\section{Conclusion and Recommendations}

Despite the limitations and challenges identified, the PHASE in Health Care Case Study Team examined the sociopolitical and economic contexts of health disparities among health care workers using a novel approach. We believe that our qualitative case study design provided a firm foundation from which to make methodological modifications that maintained rigor. The research enabled us to better understand the work environment of selected health care industry sectors in the New England region of the United States from the perspectives of multiple social actors at the micro-, meso-, and macro levels over a period of time. The final results of the study are forthcoming; however, a detailed examination of the study design is the first step in disseminating the lessons learned from this longitudinal, multisite, multidisciplinary project. Furthermore, we assert that sharing the lessons we have learned about conducting field-based, qualitative case study research of health disparities among health care workers can be useful for conducting case study health research in other dynamic workplace and community settings.

\section{Acknowledgments}

Funding

The authors disclosed receipt of the following financial support for the research and/or authorship of this article: This study was supported by the National Institute of Occupational Safety \& Health, Grant \#R01-OH07381, "Health Disparities Among Health Care Workers."

\section{Biographies}

Barbara Mawn, RN, PhD, is a professor and the director of the PhD program in nursing, and codirector of the Center for Health Promotion and Research at the University of Massachusetts Lowell in Lowell, Massachusetts, USA.

Eduardo Siqueira, MD, ScD, is an assistant professor in the Department of Community Health and Sustainability at the University of Massachusetts Lowell in Lowell, Massachusetts, USA.

Ainat Koren, RN, PhD, is an assistant professor of nursing at the University of Massachusetts Lowell in Lowell, Massachusetts, USA.

Craig Slatin, $\mathrm{ScD}, \mathrm{MPH}$, is an associate professor and chair of the Department of Community Health and Sustainability and codirector of the Center for Health Promotion and Research at the University of Massachusetts Lowell in Lowell, Massachusetts, USA. 
Karen Devereux Melillo, PhD, GNP, ANP-BC, FAANP, is a professor and the chair of Department of Nursing at the University of Massachusetts Lowell in Lowell, Massachusetts, USA.

Carole Pearce, PhD, WHNP-BC, is a professor emerita in the Department of Nursing at the University of Massachusetts Lowell in Lowell, Massachusetts, USA.

Lee Ann Hoff, RN, PhD, is the director of the Life Center Institute at the Center for Health Promotion and Research at the University of Massachusetts Lowell in Lowell, Massachusetts, USA.

\section{References}

Ashford, N., Caldart, C. Technology, law, and the working environment. New York: Van Nordstrom Rheinhold; 1991.

Austin W, Park C, Goble E. From interdisciplinary to transdisciplinary research: A case study. Qualitative Health Research. 2008; 18:557-564. [PubMed: 18354053]

Bazeley, P., Richards, L. The N Vivo qualitative project book. Thousand Oaks, CA: Sage; 2000.

Berman, D. Death on the job: Occupational health and safety struggles in the United States. New York: Monthly Review Press; 1978.

Brannon, RL. Intensifying care: The hospital industry, professionalization, and the reorganization of the nursing labor process. Amityville, NY: Baywood Publishing; 1994.

Braverman, H. Labor and monopoly degradation of work in the twentieth century. New York: Monthly Review Press; 1974.

Cheek J. Research collaboratively: Implications for qualitative research and researchers. Qualitative Health Research. 2008; 18:1599-1603. [PubMed: 18849520]

Creswell, J. Qualitative inquiry and research design: Choosing among five approaches. Thousand Oaks, CA: Sage; 2006.

Denzin, NK., Lincoln, YS. Collecting and interpreting qualitative materials. Vol. 3. Thousand Oaks, CA: Sage; 1998.

Denzin, NK., Lincoln, YS. The SAGE handbook of qualitative research. 3rd. Thousand Oaks, CA: Sage; 2005.

Eisenhardt KM. Building theories for case study research. Academy of Management Review. 1989; 14(4):532-550.

Hamel, J., Dufour, S., Fortin, D. Case study methods. Newbury Park, CA: Sage; 1993.

Jessop, B. Fordism and post-Fordism: A critical reformulation. In: Scott, AJ., Storper, MJ., editors. Pathways to regionalism and industrial development. London: Routledge; 1992. p. 43-65.

Kitzinger J. The methodology of focus groups: The importance of interaction between research participants. Sociology of Health and Illness. 1994; 16:103-121.

Krueger, RA., Casey, MA. Focus groups: A practical guide for applied research. 3rd. Thousand Oaks, CA: Sage; 2000.

Kvale, S. Interviews: An introduction to qualitative research interviewing. Thousand Oaks, CA: Sage; 1996.

Landsbergis P, Cahill J, Schnall P. The impact of lean production and related new systems of worker organization on worker health. Journal of Occupational Health Psychology. 1999; 4:108-130. [PubMed: 10212864]

Levenstein C, Tuminaro DJ. The political economy of occupational disease. New Solutions, a Journal of Environmental and Occupational Health Policy. 1991; 2:25-34.

Levenstein, C., Wooding, J., Rosenberg, B. The social context of occupational health. In: Levy, B., Wegman, D., editors. Occupational health: Recognizing and preventing work-related disease. Boston: Little, Brown and Co; 1995. p. 25-56. 
Lincoln, YS., Guba, EG. Naturalistic inquiry. Thousand Oaks, CA: Sage; 1985.

Mendlinger S, Cwikel J. Spiraling between qualitative and quantitative data on women's health behaviors: A double helix model for mixed methods. Qualitative Health Research. 2008; 18:280293. [PubMed: 18216346]

Miles, MB., Huberman, AM. Qualitative data analysis. 2nd. Thousand Oaks, CA: Sage; 1994.

Morgan, DL. Successful focus groups: Advancing the state of the art. Thousand Oaks, CA: Sage; 1993.

Morgan, DL. Focus groups as qualitative research. 2nd. Thousand Oaks, CA: Sage; 1997.

Morgan, DL. Planning focus groups. Thousand Oaks, CA: Sage; 1998.

Morse J. Serving two masters: The qualitatively driven, mixed method proposal. Qualitative Health Research. 2008; 18:1607-1608. [PubMed: 19008360]

Noble, C. Liberalism at work: The rise and fall of OSHA. Philadelphia: Temple University Press; 1986.

O'Cathain A, Murphy E, Nicholl J. Multidisciplinary, interdisciplinary, or dysfunctional? Team working in mixed-methods research. Qualitative Health Research. 2008; 18:1574-1585. [PubMed: 18849518]

Patton, MQ. Qualitative evaluation and research methods. 2nd. Newbury Park CA: Sage; 1990.

Patton MQ. Enhancing the quality and credibility of qualitative analysis. Health Services Research. 1999; 34:1189-1208. [PubMed: 10591279]

Ragin C. The distinctiveness of case-oriented research. Health Services Research. 1999; 34:11371152. [PubMed: 10591277]

Rice, PL., Ezzy, D. Qualitative research methods: A health focus. New York: Oxford University Press; 1999.

Richards, L. Using N Vivo in qualitative research. London: Sage; 1999.

Sharp K. The case for case studies in nursing research: The problem of generalization. Journal of Advanced Nursing. 1998; 27:785-789. [PubMed: 9578209]

Shin K, Kim M, Chung S. Methods and strategies utilized in published qualitative research. Qualitative Health Research. 2009; 19:850-858. [PubMed: 19429769]

Shindul-Rothschild J, Berry D, Long-Middleton E. Where have all the nurses gone? Final results of AJN's patient care survey. American Journal of Nursing. 1996; 96:25-39.

Siqueira CE. Dependent convergence: The struggle to control petrochemical hazards in Brazil and the United States (Doctoral dissertation, University of Massachusetts, Lowell, 1998). Dissertation Abstracts International. 1998; B 59/10:5331.

Slatin C. Training for action: The political economy of the Superfund Worker Training Program (Doctoral dissertation, University of Massachusetts, Lowell, 1999). Dissertation Abstracts International. 1999; B 60/05:2071.

Slatin C, Galizzi M, Melillo KD, Mawn B, The PHASE in Health Care Research Team. Conducting interdisciplinary research to promote healthy and safe employment in health care: Promises and pitfalls. Public Health Reports. 2004; 119:60-72. [PubMed: 15147650]

Sofaer S. Qualitative methods: What are they and why should we use them? Health Services Research. 1999; 34:1101-1118. [PubMed: 10591275]

Stake, RE. The art of case study research. Thousand Oaks, CA: Sage; 1995.

Stake, RE. Qualitative case studies. In: Denzin, N., Lincoln, Y., editors. The Sage handbook of qualitative research. 3rd. Thousand Oaks, CA: Sage; 2005. p. 443-466.

Thomas MD, Blacksmith J, Reno J. Utilizing insider-outsider research teams in qualitative research. Qualitative Health Research. 2000; 10:819-828. [PubMed: 11146861]

U. S. Bureau of Labor Statistics. Occupational injuries and illnesses: Counts, rate, and characteristics. Washington, DC: Author; 1997. Bulletin 2518

Weinberg, DB. Code green: Money-driven hospitals and the dismantling of nursing. Ithica, NY: Cornell University Press; 2003.

Wooding, J., Levenstein, C. The point of production. New York: Guilford; 1999.

Yin, R. Case study research: Design and methods. 2nd. Thousand Oaks, CA: Sage; 1994. 
Yin R. Enhancing the quality of case studies in health services research. Health Services Research. 1999; 34:1209-1220. [PubMed: 10591280]

Yin, R. Case study research: Design and methods. 3rd. Thousand Oaks, CA: Sage; 2003. 


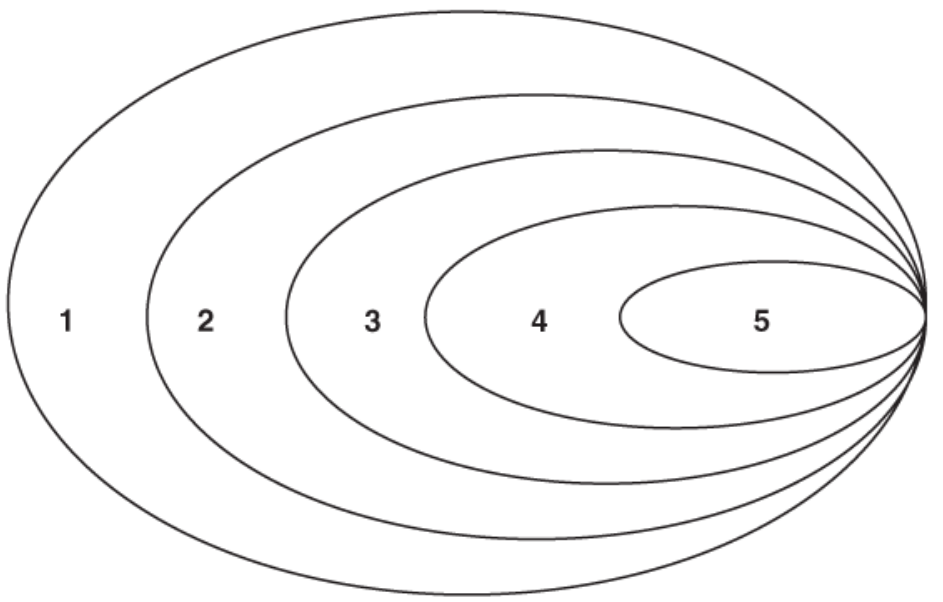

1 = macro political \& economic environment; 2 = legal \& regulatory environment; $3=$ health care environment; $4=$ organizational environment; $5=$ health care work environment

Figure 1.

Political economy of the health care environment 


\section{Table 1}

\section{Example of Labor Management Data Collection Matrix}

\begin{tabular}{|c|c|c|}
\hline Work Environment Indicators & Pertinent Questions & Potential Data Sources \\
\hline Communication & How does communication flow in the organization? & $\begin{array}{l}\text { Worker focus groups Management } \\
\text { interviews }\end{array}$ \\
\hline Employee input & $\begin{array}{l}\text { What are the procedures by which employees can raise issues of } \\
\text { concern? }\end{array}$ & $\begin{array}{l}\text { Newsletters Formal policies Media } \\
\text { reports }\end{array}$ \\
\hline Management response & How does management respond to employee concerns? & Facility reports \\
\hline Employee satisfaction & $\begin{array}{l}\text { Do employees have a sense of community? Are they satisfied with } \\
\text { management's response? }\end{array}$ & Employee surveys \\
\hline
\end{tabular}


Table 2

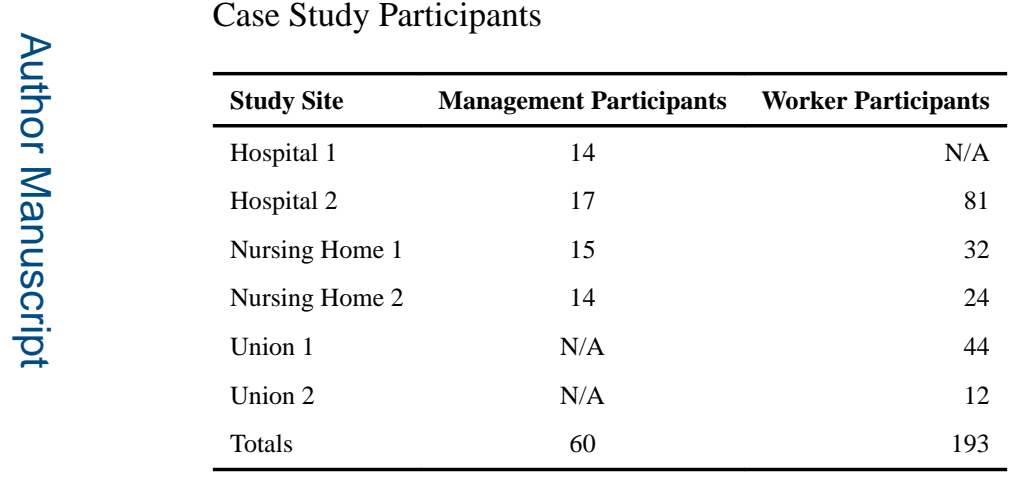

N/A = Not applicable 